\title{
Does Muscle Mass Affect Running Times in Male Long-distance Master Runners?
}

\author{
Beat Knechtle ${ }^{* 1,2}$, MD; Christoph Alexander Rüst ${ }^{1}$, BM; Patrizia Knechtle ${ }^{2}$; \\ Thomas Rosemann', MD, PhD
}

\author{
Authors' Affiliation: \\ 1. Institute of General Practice and for \\ Health Services Research, \\ University of Zurich, Zurich, \\ Switzerland \\ 2. Gesundheitszentrum St. Gallen, St. \\ Gallen, Switzerland \\ * Corresponding Author; \\ Address: Facharzt FMH für \\ Allgemeinmedizin \\ Gesundheitszentrum St. Gallen \\ Vadianstrasse 26 \\ 9001 St. Gallen \\ Switzerland \\ E-mail: beat.knechtle@hispeed.ch
}

Received: Mar 13, 2012

Accepted: Jul 19, 2012

Key Words: Body Fat; Skinfold Thickness; Anthropometry; Running; Sports

\begin{abstract}
Purpose: The aim of the present study was to investigate associations between skeletal muscle mass, body fat and training characteristics with running times in master athletes (age $>35$ years) in half-marathon, marathon and ultra-
\end{abstract} marathon.

Methods: We compared skeletal muscle mass, body fat and training characteristics in master half-marathoners $(n=103)$, master marathoners $(n=91)$ and master ultra-marathoners $(n=155)$ and investigated associations between body composition and training characteristics with race times using bi- and multi-variate analyses.

Results: After multi-variate analysis, body fat was related to half-marathon $(\beta=0.9, P=0.0003)$, marathon $(\beta=2.2, P<0.0001)$, and ultra-marathon $(\beta=10.5$, $P<0.0001)$ race times. In master half-marathoners $(\beta=-4.3, P<0.0001)$ and master marathoners $(\beta=-11.9, P<0.0001)$, speed during training was related to race times. In master ultra-marathoners, however, weekly running kilometers $(\beta=-1.6, P<0.0001)$ were related to running times.

Conclusions: To summarize, body fat and training characteristics, not skeletal muscle mass, were associated with running times in master half-marathoners, master marathoners, and master ultra-marathoners. Master half-marathoners and master marathoners rather rely on a high running speed during training whereas master ultra-marathoners rely on a high running volume during training. The common opinion that skeletal muscle mass affects running performance in master runners needs to be questioned.

Asian Journal of Sports Medicine, Volume 3 (Number 4), December 2012, Pages: 247-256

\section{INTRODUCTION}

$\mathrm{M}$ aster athletes are defined as athletes of 35 years of age and older ${ }^{[1,2]}$. These athletes maintain a high level of fitness throughout their lifespan ${ }^{[3]}$. Due to their regular training, they show only minor changes in aerobic capacity and body composition across years ${ }^{[4-}$ 6]. In recent years, several studies described an increased participation of master athletes in different running distances such as marathon ${ }^{[7,8]}$ and ultramarathon ${ }^{[9-12]}$. In recent years, master athletes improved their race performance across years in both marathons ${ }^{[7,8]}$ and ultra-marathons ${ }^{[11,12]}$.

Running performance declines with increasing age
[13-16]. The time to complete a marathon gradually increases with age, with substantial losses in performance after the age of 70 years ${ }^{[17]}$. Among the master athletes, the performance of marathoners declines after the age of $\sim 40$ years, where the peak levels of performance decreased by $\sim 50 \%$ by the age of 80 years ${ }^{[18]}$. Marathon running performance is generally fastest, as indicated by world record performances, when individuals are between 25 and 35 years old ${ }^{[17]}$. There seems to be differences regarding the age of peak running performance between distances up to the marathon and ultra-marathon distance. Halfmarathoners and marathoners can maintain their peak running times from 20 to 55 years ${ }^{[14,15]}$. In ultra- 
marathoners, however, the age of peak running performance across life is shorter and between 30 to 49 years ${ }^{[9,11,12]}$.

Factors contributing to the age-related decline in endurance performance in master athletes are central factors such as maximum heart rate, maximum stroke volume, blood volume and peripheral factors such as skeletal muscle mass, muscle fiber composition, fiber size, fiber capillarization and muscle enzyme activity ${ }^{[2]}$. The decline in endurance performance appears to be primarily due to an age-related decrease in maximum oxygen uptake $\left(\mathrm{VO}_{2} \max \right)^{[2]}$. There appears also an age-related decrease in active skeletal muscle mass contributing to a decreased endurance performance ${ }^{[2]}$.

The main reason for the age-related decline in endurance performance is the decrease in $\mathrm{VO}_{2}$ max due to a loss of skeletal muscle mass ${ }^{[19]}$. With ageing, skeletal muscle atrophy in humans appears to be inevitable. A gradual loss of muscle fibres begins at the age of $\sim 50$ years and continues to the age of $\sim 80$ years where $\sim 50 \%$ of the fibres are lost from the limb muscles ${ }^{[3]}$. The degree of atrophy of the muscle fibres is largely dependent on the habitual level of physical activity of the individual ${ }^{[3]}$. With increasing age, both $\mathrm{VO}_{2} \max { }^{[17,19-21]}$ and skeletal muscle mass ${ }^{[18,21]}$ decrease in both untrained ${ }^{[18,21]}$ and trained ${ }^{[19,20]}$ subjects. In non-endurance trained subjects, a large portion of the age-associated decline in $\mathrm{VO}_{2} \max$ is explicable by the loss of skeletal muscle mass ${ }^{[18]}$. In master athletes, an association between oxidative tissue and oxygen uptake has been described where master athletes with the greatest loss in $\mathrm{VO}_{2}$ max showed also the greatest loss in lean body mass ${ }^{[20]}$.

Apart from skeletal muscle mass, also body fat shows changes across lifetime. With increasing age, body fat increases in both untrained ${ }^{[22,23]}$ and master athletes ${ }^{[5,24,25]}$ and a greater fat mass is associated with a greater decline in leg lean mass ${ }^{[23]}$. It has also been shown that body fat is a strong predictor variable for endurance performances ${ }^{[26-30]}$ such as cycling ${ }^{[26]}$, triathlon ${ }^{[27,28,30]}$ and running ${ }^{[29]}$. In master athletes, the age-related decrease in both $\mathrm{VO}_{2} \max { }^{[5,6,19]}$ and skeletal muscle mass ${ }^{[4,5,8,17,35]}$ as well as the increase in fat mass ${ }^{[5]}$ can be partially prevented by regular physical training. In master runners, the skeletal muscle continues to have a high aerobic potential, while a decline in muscle cell size and contractile performance are apparent. These changes in the skeletal muscle profile may contribute to distance running performance decline with increasing age ${ }^{[17]}$.

To date, the age-related decline in running performance has been investigated for halfmarathoners [14,15], marathoners [14,15] and ultramarathoners ${ }^{[9,11,12]}$. For master runners, a decline in performance with age has been reported for distances up to the marathon ${ }^{[1,31,32]}$, but not for ultramarathoners. The aim of the present study was to investigate associations between skeletal muscle mass, body fat and training characteristics with running times in master athletes (age $>35$ years) in different endurance running distances such as half-marathon, marathon and ultra-marathon. We hypothesized that (i) skeletal muscle mass would decrease with increasing age, (ii) fat mass would increase with increasing age and (iii) the age-related decline in skeletal muscle mass would lead to a decrease in running times in master runners of different distances.

\section{METHODS AND SUBJECTS}

\section{Demographic and anthropometric characteristics:}

All male ultra-marathoners in the ' $100 \mathrm{~km}$ Lauf Biel' and all male runners at the 'Basel Marathon' in Basel, Switzerland, were informed, via an electronic newsletter sent by the organizer three months before the start of the race, plus separate information shown on the race website about the planned investigation. In the 'Basel Marathon', athletes can participate in both a half-marathon and a marathon. Since participation in ultra-endurance races is low per race ${ }^{[12]}$, data were collected from four consecutive years, 2008 to 2011, to increase the sample size of the $100-\mathrm{km}$ ultramarathoners. In the 'Basel Marathon' athletes were recruited in two consecutive years, from 2010 to 2011. Due to the low female participation in ultra-endurance races ${ }^{[12]}$, we focussed on male runners. Master athletes are defined as athletes with an age of $>35$ years ${ }^{[2]}$. In the '100 km Lauf Biel', 155 male master's level runners were recruited, in the 'Basel Marathon', 103 
male master's level half-marathoners and 91 male master's level marathoners were measured pre-race. Table 1 shows the age, and both the anthropometric characteristics and training variables of the subjects.

Ethical approval was granted for each event by the Institutional Review Board of the Canton of St. Gallen, Switzerland. The athletes were informed of the experimental procedures and gave their written informed consent.

\section{The Races:}

The '100 km Lauf Biel' in Biel, Berne, Switzerland, generally takes place during the night of the first weekend in June. The athletes start the ultra-marathon at 10:00 p.m. They have to climb a total altitude of 645 meters. During these $100 \mathrm{~km}$, the organizer provides a total of 17 aid stations offering an abundant variety of food and beverages such as carbohydrate-electrolyte beverage, tea, soup, carbonated cola beverage, water, bananas, oranges, bread and energy bars. The athletes are allowed to be supported by a cyclist in order to have additional food and clothing, if necessary. In the 'Basel Marathon', the athletes have to run in the marathon two laps on asphalt with a total altitude of $200 \mathrm{~m}$, in the half-marathon one lap. No athlete was included more than once and no athlete competed in either the $100-\mathrm{km}$ ultra-marathon or in the marathon.

\section{Measurements and Calculations:}

Upon inscription to the study three months before the start of both the ' $100 \mathrm{~km}$ Lauf Biel' and the 'Basel Marathon', the subjects were asked to record their training units showing duration in minutes and distance in kilometres until the start of the race. The investigator provided an electronic file where the subjects could insert each training unit with distance, duration and speed, expressed in $\mathrm{km} / \mathrm{h}$. The investigator then calculated the mean weekly hours, the mean weekly kilometres and the mean speed in running during training in the pre-race preparation.

\section{Anthropometric measurements:}

Before the start of the race, body mass, body height, the circumferences of the limbs (i.e. mid-upper arm, mid-thigh, and mid-calf) and the thicknesses of eight skin-folds (i.e. pectoralis, axillar, triceps, subscapular, abdomen, suprailiacal, thigh and calf) were measured on the right side of the body. With this data, percentage of body fat and skeletal muscle mass, using an anthropometric method, were estimated. Body mass was measured using a commercial scale (Beurer BF 15, Beurer GmbH, Ulm, Germany) to the nearest $0.1 \mathrm{~kg}$ after voiding of the urine bladder. Body height was determined using a stadiometer to the nearest $1.0 \mathrm{~cm}$ (Tanita HR 001 Portable Height Measure, Tanita Europe, Amsterdam, Netherlands). The circumferences and the lengths of the limbs were measured using a non-elastic tape measure (KaWe CE, Kirchner und Welhelm, Germany) to the nearest $0.1 \mathrm{~cm}$. The skinfold data were obtained using a skin-fold caliper (GPM-Hautfaltenmessgerät, Siber \& Hegner, Zurich, Switzerland) and recorded to the nearest $0.2 \mathrm{~mm}$. The skin-fold caliper measures with a pressure of $0.1 \mathrm{mPa} \pm$ $5 \%$ over the whole measuring range. The skin-fold

Table 1: A comparison of age, anthropometric characteristics and training between the three groups

\begin{tabular}{|c|c|c|c|}
\hline Parameter & $\begin{array}{l}\text { Half- marathoners } \\
\qquad(n=103)\end{array}$ & $\begin{array}{c}\text { Marathoners } \\
\quad(n=91)\end{array}$ & $\begin{array}{c}\text { 100-km ultra- } \\
\text { Marathoners }(n=155)\end{array}$ \\
\hline Age (years) & $45.2(7.6)$ & $47.8(7.9)$ & $47.4(7.8)$ \\
\hline Body mass (kg) & $75.9(8.7)$ & $74.1(8.5)$ & $75.1(9.5)$ \\
\hline Body height (m) & $1.78(0.06)$ & $1.77(0.05)$ & $1.78(0.06)$ \\
\hline Body mass index $\left(\mathrm{kg} / \mathrm{m}^{2}\right)$ & $23.8(2.2)$ & $23.5(2.3)$ & $23.5(2.1)$ \\
\hline Skeletal muscle mass (kg) & $38.7(3.2)$ & $37.9(3.3)$ & $38.7(3.9)$ \\
\hline Percentage of body fat $(\%)$ & $18.2(4.4)$ & $16.9(3.4)^{\#}$ & $16.4(4.3)^{\dagger, \S}$ \\
\hline Weekly running kilometers $(\mathrm{km})$ & $33.5(17.7)$ & $45.3(22.7) \#$ & $71.3(26.5)^{\dagger, \S}$ \\
\hline Weekly running hours (h) & $3.7(1.6)$ & $4.9(2.1)^{\#}$ & $7.8(4.5)^{\dagger, \S}$ \\
\hline Running speed (km/h) & $10.6(1.4)$ & $11.0(1.3)$ & $10.1(2.2)$ \\
\hline
\end{tabular}


measurements were taken following the ISAK standard once for all seven skin-folds and then the procedure was repeated twice more by the same investigator; the mean of the three measures was then used for the analyses. The timing of the taking of the skin-fold measurements was standardised to ensure reliability. According to Becque et al. ${ }^{[33]}$, readings were performed $4 \mathrm{~s}$ after applying the caliper. An intra-tester reliability check was conducted on 27 male athletes prior to testing ${ }^{[34]}$.

\section{Estimation of body fat and skeletal muscle mass:}

Percentage of body fat was estimated using the anthropometric formula according to Ball et al. ${ }^{[35]}$ for men with percentage of body fat $=$

$0.465+0.180 \times(\Sigma 7 \mathrm{SF})-0.0002406 \times(\Sigma 7 \mathrm{SF})^{2}+$ $0.0661 \times$ (age)

where $\Sigma 7 \mathrm{SF}$ is the sum of skin-fold thickness of pectoralis, axillar, triceps, subscapular, abdomen, suprailiacal, and thigh in $\mathrm{mm}$; age is in years. The predicted residual sum of squares (PRESS) $r^{2}$ was high (0.90) and the PRESS standard error of estimates (SEE) was excellent $(2.2 \%$ at the mean) for the equation when applied to a sample of 160 men. Skeletal muscle mass (kg) was estimated using the anthropometric equation of Lee et al. ${ }^{[36]}$ with skeletal muscle mass $=$

Ht $\times\left(0.00744 \times \mathrm{CAG}^{2}+0.00088 \times \mathrm{CTG}^{2}+0.00441 \times\right.$ $\left.\mathrm{CCG}^{2}\right)+2.4 \mathrm{x}$ sex $-0.048 \mathrm{x}$ age + race +7.8

where Ht: height, CAG: skin-fold-corrected upper arm girth, CTG: skin-fold-corrected thigh girth, CCG: skinfold-corrected calf girth, sex: 1 for male; age is in years; race: 0 for white men and 1 for black men. This equation was validated using magnetic resonance imagining (MRI) to determine skeletal muscle mass. There was a high correlation between the predicted skeletal muscle mass and the MRI-measured skeletal muscle mass $\left(r^{2}=0.83, P<0.0001, \mathrm{SEE}=2.9 \mathrm{~kg}\right)$. The correlation between the measured and the predicted skeletal muscle mass difference and the measured skeletal muscle mass was significant $\left(r^{2}=0.90\right.$, $P=0.009)^{[36]}$.

\section{Statistical Analyses:}

Data were checked for normal distribution and for homogeneity of variances. Data are presented as mean \pm standard deviation (SD). A one way analysis of variance (ANOVA) was used to determine differences between groups. A Tukey's post-hoc test was performed when the overall $\mathrm{F}$ value of the model was significant to detect differences. To investigate a potential association between anthropometric characteristics, training variables and race time, in a first step, Pearson correlation analysis was used to check for associations between parameters. For the strength of a correlation, $r>0.70$ indicated a very strong, $r=0.40$ to 0.69 a strong, $r=0.30$ to 0.39 a moderate, $r=0.20$ to 0.29 a weak and $r=0.01$ to 0.19 a negligible relationship, respectively. Since also other variables than skeletal muscle mass such as body fat and training variables are also related to running times [29], we performed, in a second step, multi-variate analyses to correct the association of skeletal muscle mass, body fat and training variables with race time with co-variates. In this second step, all significant variables of bi-variate analysis entered the multiple linear regression analysis (stepwise, forward selection, $P$ of $\mathrm{F}$ for inclusion $\leq 0.05, P$ of $\mathrm{F}$ for exclusion $\geq 0.1$ ) Multicollinearity between the predictor variables was excluded with $r>0.9$. Statistical significance was accepted with $P<0.05$ (two-sided hypothesis).

\section{RESULTS}

\section{Comparison between groups:}

The master half-marathoners, master marathoners and master ultra-marathoners showed no differences regarding their age, body mass, body height and body mass index $(P>0.05)$ (Table 1). Skeletal muscle mass was not different between the groups $(P>0.05)$. Percentage of body fat was lowest in the $100-\mathrm{km}$ master ultra-marathoners $(P<0.05)$. Weekly running kilometers and weekly running hours were highest in the $100-\mathrm{km}$ master ultra-marathoners $(P<0.05)$. Running speed during training sessions was not different between the groups $(P>0.05)$.

\section{Association between age and anthropometry:}

Age was moderately significant and negatively related 
to skeletal muscle mass for master half-marathoners $(r=-0.31, P=0.002)$ and master marathoners $(r=-0.38$, $P=0.0002)$. For master ultra-marathoners, age was strongly significant and negatively related to skeletal muscle mass $(r=-0.53, P<0.0001)$. In master halfmarathoners $(r=0.22, P=0.03)$, master marathoners ( $r=0.24, \quad P=0.02)$, and master ultra-marathoners ( $r=0.23, P=0.005)$, age was weakly significant and positively related to percentage of body fat. The coefficient of correlation between age and skeletal muscle mass decreased with increasing length of the running distance (Fig. 1).

For the correlation between age and percentage of body fat, the coefficient of correlation remained unchanged for all three running distances (Fig. 1).

\section{Association of pre-race anthropometry with training:}

Skeletal muscle mass showed no association with training variables $(P>0.05)$. Percentage of body fat was moderately significant and negatively related to weekly running kilometers $(r=-0.31, P=0.001)$ and running speed during training sessions $(r=-0.33, P=0.0007)$ in master half-marathoners. In master marathoners, percentage of body fat was weakly significant and negatively related to weekly running kilometers ( $r=-$ 0.26, $P=0.01$ ) and moderately significant and negatively to running speed during training sessions ( $r=-0.34, \quad P=0.001)$. In master ultra-marathoners, percentage of body fat was moderately significant and negatively related to weekly running kilometers ( $r=$ $0.32, P<0.0001)$ and weakly significant and negatively to running speed during training sessions $(r=-0.25$, $P=0.001)$.

\section{Association of age and anthropometry with running times:}

Age was weakly significant and positively $(r=0.27$, $P=0.005)$ related to race time in master halfmarathoners, and moderately significant in master marathoners $(r=0.33, P=0.001)$ and master ultramarathoners $(r=0.37, P<0.0001)$ where the coefficients of correlation increased with increasing running distance. Skeletal muscle mass showed no relationship with race time $(P>0.05)$. Percentage of body fat was
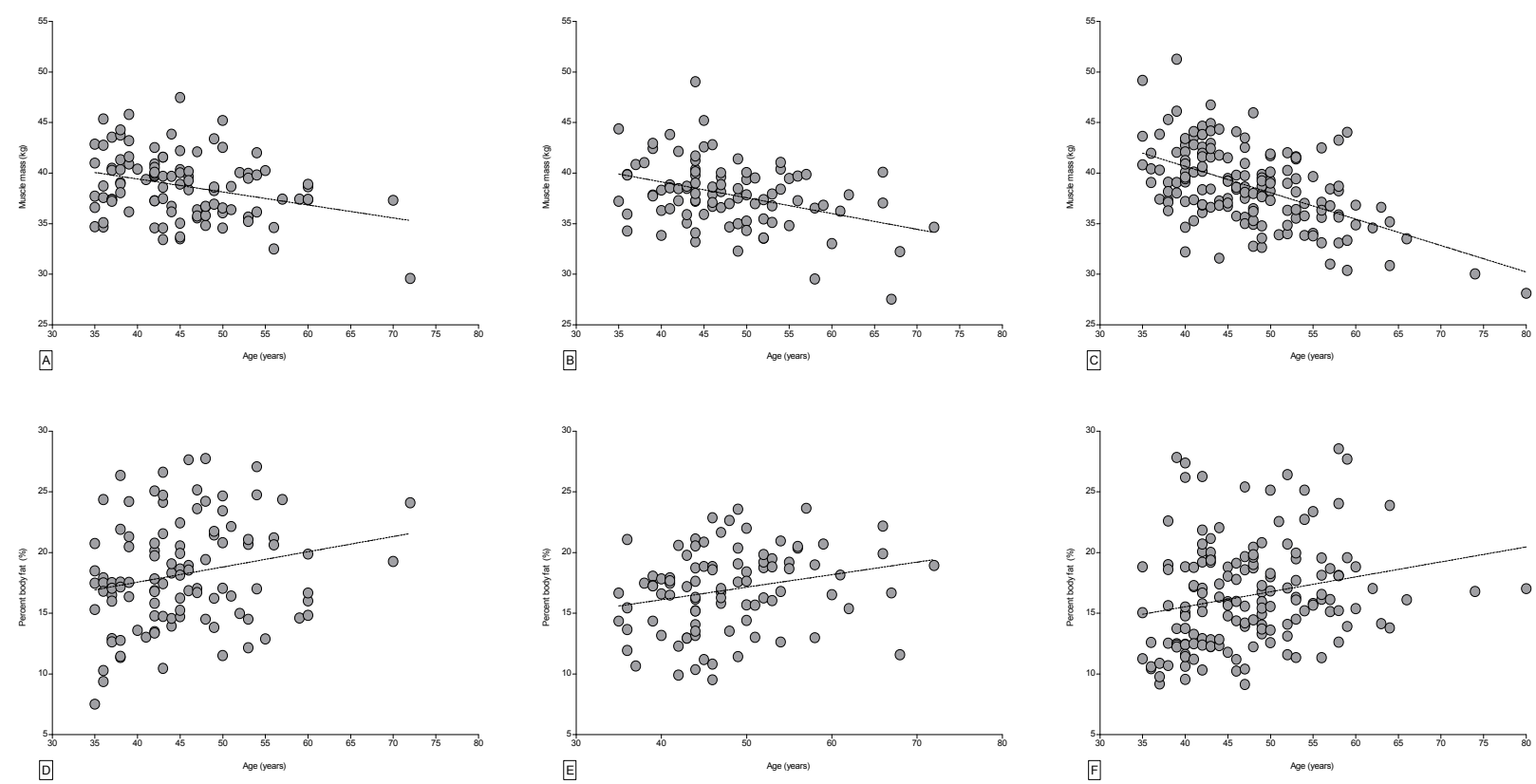

Fig. 1: The association of skeletal muscle mass with age for master half-marathoners $(r=-0.31)$ (Panel A), for master marathoners $(r=-0.38)$ (Panel B) and 100-km master ultra-marathoners $(r=-0.53)$ (Panel C). With increasing length of the running performance, the coefficient of correlation became more negative. For percentage of body fat, the association with age 
for master half-marathoners $(r=0.22)$ (Panel D), master marathoners $(r=0.24)$ (Panel E) and 100-km master ultra-marathoners $(r=0.23)$ (Panel F) showed the same coefficient of correlation

strongly significant and positively related to race times in master half-marathoners $(r=0.45, P<0.0001)$, master marathoners $(r=0.48, P<0.0001)$ and master ultramarathoners $(r=0.48, P<0.0001)$ where the coefficients of correlation also increased with increasing running distance.

\section{Association of training with running times:}

In master half-marathoners, weekly running hours were weakly significant and negatively $\operatorname{related}(r=-0.28$, $P=0.004)$, weekly running kilometers $(r=-0.48$, $P<0.0001)$ and running speed during training sessions ( $r=-0.57, \quad P<0.0001$ ) however, were strongly significant and negatively related to race time. In master marathoners, weekly running hours were not related to race time $(P>0.05)$. Weekly running kilometres were weakly significant and negatively ( $r=-0.25, P=0.01$ ) and running speed during training was strongly significant and negatively related $(r=-0.64, P<0.0001)$ related to race time. For master ultra-marathoners, weekly running hours were negligibly significant and negatively $(r=-0.18$, $P=0.02$ ), weekly running kilometres strongly significant and negatively $(r=-0.48, P<0.0001)$ and running speed during training weakly significant and negatively ( $r=-0.28, P=0.0004)$ related to race times.

\section{Multi-variate analyses:}

In the multi-variate analysis (Table 2$)$, age $(P<0.0001)$, skeletal muscle mass $(P=0.008)$, body fat $(P=0.0001)$ and running speed during training $(P<0.0001)$ were related to running times in master ultra-marathoners. For master half-marathoners and master marathoners, however, neither age nor skeletal muscle mass was related to running times $(P>0.05)$. For both master half-marathoners and master marathoners, percentage of body fat $(P=0.008$ and $P=0.004$, respectively) and running speed during training $(P<0.0001)$ were related to running times whereas in master half-marathoners, also weekly running kilometers $(P=0.01)$ were associated with running times. When the age variable was removed from the multi-variate models (Table 3 ), body fat was related to half-marathon $(P=0.0003)$, marathon $(P<0.0001)$, and ultra-marathon $(P<0.0001)$ running times.

In master half-marathoners $(P<0.0001)$ and master marathoners $(P<0.0001)$, speed during training was related to running times. In master ultra-marathoners,

Table 2: Associations between significant characteristics after bi-variate analysis and race time for the master halfmarathoners, master marathoners and 100-km master ultra-marathoners using multiple linear regression analysis

\begin{tabular}{|c|c|c|c|c|}
\hline Group & & $\beta$ & SE & $P$ Value \\
\hline \multirow{6}{*}{$\begin{array}{l}\text { Half-marathoners } \\
(n=103)\end{array}$} & Age & 0.3 & 0.2 & 0.1 \\
\hline & Skeletal muscle mass & -0.2 & 0.4 & 0.7 \\
\hline & Percentage of body fat & 0.9 & 0.3 & 0.008 \\
\hline & Weekly running hours & 0.1 & 1.1 & 0.9 \\
\hline & Weekly running kilometres & -0.3 & 0.1 & 0.01 \\
\hline & Speed in running training & -4.5 & 1.0 & $<0.0001$ \\
\hline \multirow{6}{*}{$\begin{array}{l}\text { Marathoners } \\
(n=91)\end{array}$} & Age & 0.6 & 0.3 & 0.06 \\
\hline & Skeletal muscle mass & 1.2 & 0.8 & 0.1 \\
\hline & Percentage of body fat & 2.2 & 0.7 & 0.004 \\
\hline & Weekly running hours & -0.5 & 1.8 & 0.8 \\
\hline & Weekly running kilometres & -0.05 & 0.2 & 0.7 \\
\hline & Speed in running training & -11.6 & 1.9 & $<0.0001$ \\
\hline \multirow{4}{*}{$\begin{array}{l}100 \text {-km ultra- } \\
\text { marathoners } \\
(n=155)\end{array}$} & Age & 6.4 & 1.2 & $<0.0001$ \\
\hline & Skeletal muscle mass & 6.2 & 2.3 & 0.008 \\
\hline & Percentage of body fat & 7.6 & 1.9 & 0.0001 \\
\hline & Weekly running hours & -2.1 & 1.9 & 0.3 \\
\hline
\end{tabular}




$\begin{array}{lllc}\text { Weekly running kilometres } & -4.8 & 3.9 & 0.2 \\ \text { Speed in running training } & -1.5 & 0.3 & <0.0001\end{array}$

$\beta=$ regression coefficient; $S E=$ standard error of the regression coefficient. The coefficient of determination $\left(r^{2}\right)$ of the model was 0.47 for master half-marathoners, 0.55 for master marathoners, and 0.47 for master ultra-marathoners, respectively

Table 3: Associations between significant characteristics after bi-variate analysis and race time for the master half-marathoners, master marathoners and 100-km master ultra-marathoners using multiple linear regression analysis after removing the age variable

\begin{tabular}{|c|c|c|c|c|}
\hline Group & & $\beta$ & $S E$ & $P$ Value \\
\hline \multirow{5}{*}{$\begin{array}{l}\text { Half-marathoners } \\
(n=103)\end{array}$} & Skeletal muscle mass & -0.3 & 0.3 & 0.3 \\
\hline & Percentage of body fat & 0.9 & 0.3 & 0.0003 \\
\hline & Weekly running hours & 0.6 & 0.8 & 0.4 \\
\hline & Weekly running kilometres & -0.3 & 0.08 & 0.002 \\
\hline & Speed in running training & -4.3 & 0.8 & $<0.0001$ \\
\hline \multirow{5}{*}{$\begin{array}{l}\text { Marathoners } \\
(n=91)\end{array}$} & Skeletal muscle mass & 0.9 & 0.7 & 0.2 \\
\hline & Percentage of body fat & 2.2 & 0.6 & 0.0008 \\
\hline & Weekly running hours & 0.8 & 1.3 & 0.5 \\
\hline & Weekly running kilometres & -0.2 & 0.1 & 0.2 \\
\hline & Speed in running training & -11.9 & 1.7 & $<0.0001$ \\
\hline \multirow{5}{*}{$\begin{array}{l}\text { 100-km ultra-marathoners } \\
(n=155)\end{array}$} & Skeletal muscle mass & -0.7 & 2.1 & 0.8 \\
\hline & Percentage of body fat & 10.5 & 2.0 & $<0.0001$ \\
\hline & Weekly running hours & 0.05 & 2.0 & 0.9 \\
\hline & Weekly running kilometres & -1.6 & 0.4 & $<0.0001$ \\
\hline & Speed in running training & -3.0 & 4.2 & 0.5 \\
\hline
\end{tabular}

$\beta=$ regression coefficient; $S E=$ standard error of the regression coefficient. The coefficient of determination $\left(r^{2}\right)$ of the model was 0.48 for master half-marathoners, 0.45 for master marathoners, and 0.36 for master ultra-marathoners, respectively

however, weekly running kilometers $(P<0.0001)$ were related to ultra-marathon running times.

\section{DISCUSSION}

The main findings of this study were (i) age was significantly and negatively related to skeletal muscle mass and significantly and positively to percentage of body fat for master runners of all distances, (ii) skeletal muscle mass was not related to training characteristics whereas percentage of body fat was related to both volume and running speed during training in master runners of all distances, and (iii) percentage of body fat and training characteristics, not skeletal muscle mass, were related to running times for master runners of all distances.

\section{Age, anthropometry and training:}

Skeletal muscle mass was not different between the three groups. In all three groups of runners, skeletal muscle mass decreased with increasing age, as hypothesized. The association between skeletal muscle mass and age became more important with increasing length of a running distance. This was also backed up in the multi-variate analyses where both age and skeletal muscle mass were predictor variables for race time in the master ultra-marathoners, but neither in the master half-marathoners nor in the master marathoners. The age-related decrease in skeletal muscle mass can be partially prevented by regular physical training in master athletes ${ }^{[4,5,8,17,35]}$. Since neither age nor skeletal muscle mass were different between the three groups, we expected to find an association between training variables and skeletal muscle mass in the master ultramarathoners since they showed differences in the predictor variables. Although training volume increased with increasing race distance, we found no associations between training variables and skeletal muscle mass in these three groups of runners.

In contrast to skeletal muscle mass, percent body was different between the three groups. Percentage of body fat was lowest in the ultra-marathoners, significantly different to both the half-marathoners and the marathoners. With increasing length of the running distance, percentage of body fat became lower in the 
subjects. The lower body fat in ultra-marathoners might be due to their training. For half-marathoners ${ }^{[38]}$, marathoners ${ }^{[40]}$ and $100-\mathrm{km}$ ultra-marathoners ${ }^{[37]}$, an association between body fat and training characteristics has been shown. However, correlation analysis does not prove cause and effect and low body fat in runners might also be due to genetics or diet. Also, percentage of body fat was predictive for both half-marathoners ${ }^{[38]}$ and marathoners ${ }^{[40]}$, but not for 100-km ultra-marathoners ${ }^{[37]}$. Most probably, training characteristics are more important for ultramarathoners, since age, running speed during training and weekly running kilometers predicted $100-\mathrm{km}$ race times ${ }^{[37]}$, but not body fat. In all three groups of the present subjects, percentage of body fat increased with increasing age, as hypothesized. In contrast to skeletal muscle mass, we found significant associations between body fat and training variables in all three groups. Also for body fat, the age-related increase in fat mass can be partially prevented by regular physical training in master athletes ${ }^{[5]}$. Indeed, Legaz and Eston showed that intense training resulted in a significant increase in performance and decreases in sum of six skinfolds, abdominal, front thigh, and medial calf skinfolds ${ }^{[39]}$.

\section{Association of age, anthropometry and training with running times:}

The three samples of master's level runners showed no differences regarding their age and anthropometry. Age was significantly related to race times in all three groups after bi-variate analyses, but only for the master ultra-marathoners after multi-variate analyses. Age seems to be predictive only for running distances longer than a marathon ${ }^{[36,37]}$. A recent study showed that $100-\mathrm{km}$ ultra-marathon race time might be predicted by an equation using running speed during training, running volume and age ${ }^{[37]}$. For halfmarathoners ${ }^{[38]}$ and marathoners ${ }^{[40]}$, however, age was not predictive for race time.

Based upon existing literature it was hypothesized that the decrease in skeletal muscle mass with increasing age would be associated with slower running times in master runners. Skeletal muscle mass was not related to half-marathon and marathon running times, but to ultra-marathon running times after multi- variate analysis. When the variable 'age' was excluded from the multi-variate model, only body fat was related to running times for all three groups. Therefore, skeletal muscle mass seemed not to affect running times in master runners, independent of the running distance. However, body fat was related to running times in master half-marathoners, master marathoners and master ultra-marathoners in contrast to skeletal muscle mass. The coefficient of correlation between body fat and race times increased from $r=0.45$ for half-marathoners to $r=0.48$ for marathoners and $r=$ 0.49 for ultra-marathoners, respectively. It seemed that low body fat become more important with increasing length of a running distance. Body fat seems to be the most important anthropometric variable in endurance athletes as has already been shown for halfmarathoners ${ }^{[38]}$, marathoners ${ }^{[40]}$ and triathletes ${ }^{[26-28]}$.

An important finding was that volume and intensity in training showed differences regarding the length of the running distance. In master half-marathoners and master marathoners, speed during training was related to race times. In master ultra-marathoners, however, weekly running kilometers were related to ultramarathon running times. Obviously, master athletes prepare specifically for the length of the running distance where ultra-marathoners invest more on volume compared to marathoners investing more on intensity. The same has been reported for long-distance triathletes. Triple Iron ultra-triathletes covering 11.4 $\mathrm{km}$ swimming, $540 \mathrm{~km}$ running and $126.6 \mathrm{~km}$ running relied more on training volume in cycling and running, whereas speed in cycling training was related to race time in Ironman triathletes competing over $3.8 \mathrm{~km}$ swimming, $180 \mathrm{~km}$ cycling and $42.195 \mathrm{~km}$ running ${ }^{[30]}$. Also when marathoners and 100-km ultra-marathoners were compared, marathoners rely more on speed in running during training whereas ultra-marathoners rely on volume in running training ${ }^{[41]}$.

\section{Limitations and implications for future research:}

Skeletal muscle mass and body fat were estimated using an anthropometric equation. Both skeletal muscle mass and percentage of body fat might be more precisely determined using dual energy X-ray absorptiometry (DXA) and magnetic resonance imaging (MRI) ${ }^{[42]}$. The self-reporting of times and 
distances in training is a limitation since we have no way of establishing the reliability and precision of reporting. For future research, the reliability of training data might be enhanced by quantifying and validating self-reported training data with the use of global positioning system (GPS). Future studies need to investigate whether runners move to longer distances as they lose running speed with increasing age.

\section{CONCLUSION}

To summarize, body fat and training characteristics, not skeletal muscle mass, were associated with running times in master half-marathoners, master marathoners, and master ultra-marathoners. Master half-marathoners and master marathoners rely rather on high running speed during training whereas master ultra-marathoners rely on high running volume during training. The common opinion that skeletal muscle mass affects running performance in master runners needs to be questioned.

\section{ACKNOWLEDGMENTS}

The authors would like to thank the race directors for their help to conduct the study. A special thank goes to all our subjects.

\section{Conflict of interests: None}

\section{REFERENCES}

1. Michaelis I, Kwiet A, Gast U, et al. Decline of specific peak jumping power with age in master runners. J Musculoskelet Neuronal Interact 2008;8:64-70.

2. Reaburn P, Dascombe B. Endurance performance in masters athletes. Eur Rev Aging Phys Act. 2008;5:31-42.

3. Faulkner JA, Larkin LM, Claflin DR, Brooks SV. Age-related changes in the structure and function of skeletal muscles. Clin Exp Pharmacol Physiol 2007;34:1091-6.

4. Pollock ML, Mengelkoch LJ, Graves JE, et al. Twenty-year follow-up of aerobic power and body composition of older track athletes. J Appl Physiol 1997;82:1508-16.

5. Pollock ML, Foster C, Knapp D, et al. Effect of age and training on aerobic capacity and body composition of master athletes. $J$ Appl Physiol 1987;62:725-31.

6. Rogers MA, Hagberg JM, Martin WH 3rd, et al. Decline in VO2max with aging in master athletes and sedentary men. $J$ Appl Physiol 1990;68:2195-9.

7. Jokl P, Sethi PM, Cooper AJ. Master's performance in the New York City Marathon 1983-1999. Br J Sports Med 2004;38:408-12.

8. Lepers R, Cattagni T. Do older athletes reach limits in their performance during marathon running? Age (Dordr) 2012;34:773-81.

9. Hoffman MD. Performance trends in 161-km ultramarathons. Int J Sports Med 2010;31:31-7.

10. Hoffman MD, Ong JC, Wang G.Historical analysis of participation in $161 \mathrm{~km}$ ultramarathons in North America. Int J Hist Sport 2010;27:1877-91.

11. Hoffman MD, Wegelin JA. The Western States 100-Mile Endurance Run: participation and performance trends. Med Sci Sports Exerc 2009;41:2191-8.

12. Knechtle B, Rüst CA, Rosemann T, Lepers R. Age-related changes in 100-km ultra-marathon running performance. Age (Dordr) 2012;34:1033-45.

13. Celie F, Faes M, Hopman M, Stalenhoef AF, Rikkert MG. Running on age in a 15-km road run: minor influence of age on performance. Eur Rev Aging Phys Act 2010;7:43-47.

14. Leyk D, Erley O, Ridder D, et al. Age-related changes in marathon and half-marathon performances. Int J Sports Med 2007;28:513-7.

15. Leyk D, Erley O, Gorges W, et al. Performance, training and lifestyle parameters of marathon runners aged 20-80 years: results of the PACE-study. Int J Sports Med 2009;30:360-5.

16. Wright VJ, Perricelli BC. Age-related rates of decline in performance among elite senior athletes. Am J Sports Med 2008;36:443-50. 
17. Trappe S. Marathon runners: how do they age? Sports Med 2007;37:302-5.

18. Fleg JL, Lakatta EG. Role of muscle loss in the age-associated reduction in VO2max. J Appl Physiol 1988;65:1147-51.

19. Trappe SW, Costill DL, Vukovich MD, et al. Aging among elite distance runners: a 22-yr longitudinal study. J Appl Physiol 1996;80:285-90.

20. Hawkins SA, Marcell TJ, Victoria Jaque S, Wiswell RA. A longitudinal assessment of change in VO2max and maximal heart rate in master athletes. Med Sci Sports Exerc 2001;33:1744-50.

21. Sugawara J, Miyachi M, Moreau KL, et al. Age-related reductions in appendicular skeletal muscle mass: association with habitual aerobic exercise status. Clin Physiol Funct Imaging 2002;22:169-72.

22. Horber FF, Gruber B, Thomi F, et al. Effect of sex and age on bone mass, body composition and fuel metabolism in humans. Nutrition 1997;13:524-34.

23. Koster A, Ding J, Stenholm S, et al. Health ABC study. Does the amount of fat mass predict age-related loss of lean mass, muscle strength, and muscle quality in older adults? J Gerontol A Biol Sci Med Sci 2011;66:888-95.

24. Horber FF, Kohler SA, Lippuner K, Jaeger P. Effect of regular physical training on age-associated alteration of body composition in men. Eur J Clin Invest 1996;26:279-85.

25. Wroblewski AP, Amati F, Smiley MA, et al. Chronic exercise preserves lean muscle mass in masters athletes. Phys Sportsmed 2011;39:172-8.

26. Knechtle B, Wirth A, Knechtle P, Rosemann T. Moderate association of anthropometry, but not training volume, with race performance in male ultraendurance cyclists. Res Q Exerc Sport 2009;80:563-8.

27. Knechtle B, Wirth A, Baumann B, et al. Personal best time, percentage of body fat, and training are differently associated with race time for male and female ironman triathletes. Res Q Exerc Sport 2010;81:62-8.

28. Knechtle B, Knechtle P, Rosemann T. Upper body skinfold thickness is related to race performance in male Ironman triathletes. Int $J$ Sports Med 2011;32:20-7.

29. Knechtle B, Knechtle P, Rosemann T, Senn O. What is associated with race performance in male 100-km ultra-marathoners-anthropometry, training or marathon best time? J Sports Sci 2011;29:571-7.

30. Knechtle B, Knechtle P, Rüst CA, Rosemann T. A comparison of anthropometric and training characteristics of Ironman triathletes and Triple Iron ultra-triathletes. J Sports Sci 2011;29:1373-80.

31. Ransdell LB, Vener J, Huberty J. Masters athletes: an analysis of running, swimming and cycling performance by age and gender. $J$ Exerc Sci Fit 2009;7:61-73.

32. Rittweger J, di Prampero PE, Maffulli N, Narici MV. Sprint and endurance power and ageing: an analysis of master athletic world records. Proc Biol Sci 2009;276:683-9.

33. Becque MD, Katch VL, Moffatt RJ. Time course of skin-plus-fat compression in males and females. Hum Biol 1986;58:33-42.

34. Knechtle B, Joleska I, Wirth A, et al. Intra- and inter-judge reliabilities in measuring the skin-fold thicknesses of ultra runners under field conditions. Percept Mot Skills 2010;111:105-6.

35. Ball SD, Altena TS, Swan PD. Comparison of anthropometry to DXA: a new prediction equation for men. Eur J Clin Nutr 2004;58:1525-31

36. Lee RC, Wang Z, Heo M, et al.Total-body skeletal muscle mass: development and cross-validation of anthropometric prediction models. Am J Clin Nutr 2000;72:796-803.

37. Knechtle B, Knechtle P, Rosemann T, Lepers R. Predictor variables for a 100-km race time in male ultra-marathoners. Percept Mot Skills 2010;111:681-93.

38. Rüst CA, Knechtle B, Knechtle P, et al. Predictor variables for a half marathon race time in recreational male runners. Open Access $J$ Sports Med 2011;2:113-9.

39. Legaz A, Eston R. Changes in performance, skinfold thicknesses, and fat patterning after three years of intense athletic conditioning in high level runners. Br J Sports Med 2005;39:851-6.

40. Barandun U, Knechtle B, Knechtle $\mathrm{P}$, et al. Running speed during training and percentage of body fat predict race time in recreational male marathoners. Open Access J Sports Med 2012;3:51-8.

41. Rüst CA, Knechtle B, Knechtle P, Rosemann T. Similarities and differences in anthropometry and training between recreational male 100-km ultra-marathoners and marathoners. J Sports Sci 2012;30:1249-57.

42. Wang JG, Zhang Y, Chen HE, Li Y, et al. Comparison of two bioelectrical impedance analysis devices with dual energy X-ray absorptiometry and magnetic resonance imaging in the estimation of body composition. J Strength Cond Res 2012 Feb 15 . [Epub ahead of print] 